\title{
Cyclosporin population pharmacokinetics in pediatric refractory nephrotic syndrome based on real-world studies: Effects of body weight and spirolactone administration
}

\author{
DONGDONG WANG $^{1 *}, \mathrm{XIAO} \mathrm{CHEN}^{2 *}$ and ZHIPING LI ${ }^{1}$ \\ ${ }^{1}$ Department of Pharmacy, Children's Hospital of Fudan University, Shanghai 201102; \\ ${ }^{2}$ Department of Pharmacy, People's Hospital of Jiangyin, Jiangyin, Jiangsu 214400, P.R. China
}

Received July 8, 2018; Accepted February 4, 2019

DOI: $10.3892 /$ etm.2019.7325

\begin{abstract}
Different models of population pharmacokinetics (PPK) of cyclosporin have been established in various populations. However, the cyclosporin PPK model in patients with pediatric refractory nephrotic syndrome (PRNS) has yet to be constructed. The present study aimed to establish the cyclosporin PPK model in PRNS, and to identify factors that may account for any variability. Chinese patients with PRNS treated with cyclosporin between June 2014 and June 2018 at the Children's Hospital of Fudan University (Shanghai, China) were retrospectively analyzed. The impact of demographic features, laboratory parameters and concomitant medications was evaluated. A total of 18 PRNS patients from real-world studies were analyzed by non-linear mixed-effects modeling. A one-compartment model with first-order absorption and elimination was selected as the appropriate model in PRNS. Body weight (WT) and spirolactone intake were included as significant covariates for the apparent oral clearance (CL/F), and the WT was revealed to significantly influence the apparent volume of distribution $(\mathrm{V} / \mathrm{F})$. The final covariate models were as follows: $\mathrm{CL} / \mathrm{F}=80.7 \times(\mathrm{WT} / 70)^{0.75} \times\left(1-0.265 \times \theta_{\text {spirolactone }}\right)$, and $\mathrm{V} / \mathrm{F}=2,030 \times(\mathrm{WT} / 70)$, where $\theta_{\text {spirolactone }}$ is the coefficient of spirolactone. The inter-individual variability in $\mathrm{CL} / \mathrm{F}$ and $\mathrm{V} / \mathrm{F}$ was 44.6 and $53.1 \%$, respectively. In conclusion, in the present study, a cyclosporin PPK model for patients with PRNS was successfully constructed, and the presence of a clinically significant interaction between spirolactone and cyclosporin in PRNS patients was determined based on real-world studies,
\end{abstract}

Correspondence to: Professor Zhiping Li, Department of Pharmacy, Children's Hospital of Fudan University, 399 Wanyuan Road, Shanghai 201102, P.R. China

E-mail: zplifudan@126.com

${ }^{*}$ Contributed equally

Key words: cyclosporin, population pharmacokinetics, pediatric refractory nephrotic syndrome, real-world study, weight, spirolactone indicating that concomitant medication with spirolactone was able to reduce cyclosporin clearance in the patients with PRNS.

\section{Introduction}

Nephrotic syndrome (NS) is the most common type of glomerulopathy in pediatric patients (1). Although the majority of patients with pediatric NS have steroid-sensitive NS (SSNS), $\sim 20 \%$ of them do not achieve complete remission and the condition develops into steroid-resistant NS (SRNS) (2). An estimated $80-90 \%$ of the pediatric patients with SSNS undergo relapse, and among those, $50 \%$ relapse frequently; thereby, the condition turns into steroid-dependent NS (SDNS) (3-5). Hence, suitable therapies for pediatric refractory NS (PRNS), which includes SDNS and SRNS, represent a challenge.

Cyclosporin therapy for patients with PRNS has been described in previous studies (6-8). However, cyclosporin has a narrow therapeutic window, and also displays considerable inter- and intra-individual variabilities in terms of its pharmacokinetics (9), making it difficult to define an optimal dose and regimen for PRNS treatment.

It is encouraging that, with population pharmacokinetics (PPK), PK information may be acquired from sparse data pooled into a large group of subjects. In addition, population analysis methodology differentiates between inter-individual variability and residual unexplained variability, having greater statistical power to verify the effects of multiple factors on PK behavior, thereby making it possible to formulate an optimal dosing and administration schedule (10). At present, a small number of cyclosporin PPK models have been established in various populations $(9,11,12)$; however, the cyclosporin PPK model in patients with PRNS has yet to be constructed. The present study aimed to establish a cyclosporin PPK model for patients with PRNS, and to distinguish factors that may explain for any variability identified.

\section{Materials and methods}

Patients and data collection. All data of the present study were derived from real-world studies between June 2014 and June 2018 from the Children's Hospital of Fudan University (Shanghai, China). Patients aged $<16$ years who were diagnosed 
with PRNS and who were receiving cyclosporin therapy were selected for the present study. Patients were excluded if they had also been diagnosed with any other serious disease, including kidney failure (Fig. 1). Relevant clinical information and drug concentration data were collected from medical records and therapeutic drug monitoring (TDM) records, respectively. The present retrospective analysis was approved by the ethics committee of the Children's Hospital of Fudan University (Shanghai, China) without the requirement for written informed consent, since the data were collected without patient identifiers $(13,14)$.

Information extracted from the medical records included the following: Sex, age, body weight (WT), body height, levels of albumin, globulin, albumin/globulin, alanine transaminase, aspartate transaminase, creatinine, urea, total protein and total bile acid, direct bilirubin, total bilirubin, hematocrit, hemoglobin count, mean corpuscular hemoglobin count, mean corpuscular hemoglobin concentration and drugs that the patients may have been receiving concomitantly with cyclosporin (namely, diltiazem, dipyridamole, felodipine, fosinopril, methylprednisolone, nifedipine, piperazine ferulate, prednisolone or spirolactone).

Drug administration. All patients were orally administered cyclosporin (in liquid solution). The dose of cyclosporin administered was initially $25-80 \mathrm{mg}$ daily, although the cyclosporin dose was later adjusted according to the clinical efficacy and adverse events experienced by the patient, as well as its trough concentration in TDM.

Analytical method. Whole-blood concentrations of cyclosporin were measured using an Emit ${ }^{\circledR} 2000$ Cyclosporine Specific assay (6R079UL; Siemens Healthcare Diagnostics, Inc., Newark, NJ, USA), according to the manufacturer's protocol.

PPK modeling. Data were analyzed according to the non-linear mixed-effects model (NONMEM) computer program (version VII; ICON Development Solutions, Elicott City, MD, USA). The 'first-order conditional estimation method with interaction' option was used to estimate the PK parameters and their variability. A one-compartment model with first-order elimination was used to describe the absorption phase, as all the cyclosporin concentrations featured in the present analysis were trough concentrations. It was not possible to estimate the bioavailability (F) and absorption with a lag time, since cyclosporin was orally administered and its concentration data were insufficient. Therefore, the PK parameters studied were the apparent oral clearance $(\mathrm{CL} / \mathrm{F})$ and the apparent volume of distribution $(\mathrm{V} / \mathrm{F})$. The absorption rate constant $(\mathrm{Ka})$ of the model was fixed to $0.681 / \mathrm{h}$ according to a previous study (9).

Random-effects model. The interindividual variability in the PK parameters was explored using an exponential error model: $\mathrm{P}_{\mathrm{i}}=\mathrm{T}(\mathrm{P}) \mathrm{x} \exp \left(\eta_{\mathrm{i}}\right)$, where $\mathrm{P}_{\mathrm{i}}$ is the individual parameter value, and $T(P)$ is the typical individual parameter value. $\eta_{i}$ is the symmetrical distribution, which are zero-mean chance variables with the variance term. The residual error variability was evaluated with the mixed-error model, $\mathrm{OB}=\mathrm{IP} \times\left(1+\varepsilon_{1}\right)+\varepsilon_{2}$, where $\mathrm{OB}$ is the observation, and IP represents the individual predicted concentration. $\varepsilon_{n}$ represents the symmetrical distribution, which are zero-mean chance variables with a variance.

Covariate model. The association between WT and the PK parameters was modeled according to the following equation: $\mathrm{P}_{\mathrm{i}}=\mathrm{P}_{\text {std }} \mathrm{x}\left(\mathrm{WT}_{\mathrm{i}} / \mathrm{WT}_{\text {std }}\right)^{\mathrm{PWR}}$, where $\mathrm{P}_{\mathrm{i}}$ is the $\mathrm{PK}$ parameter of the ith individual, $\mathrm{WT}_{\mathrm{i}}$ is the body weight of the ith individual and $\mathrm{P}_{\text {std }}$ is the parameter of an individual with a WT, $\mathrm{WT}_{\text {std }}$, of $70 \mathrm{~kg}$. PWR is the allometric coefficient: 0.75 for the CL/F and 1 for the $\mathrm{V} / \mathrm{F}(15)$.

To explain the variability of the PK parameters, the potential covariates included sex, age, WT, body height, levels of albumin, globulin, albumin/globulin, alanine transaminase, aspartate transaminase, creatinine, urea, total protein, total bile acid, direct bilirubin, total bilirubin, the hematocrit, hemoglobin count, mean corpuscular hemoglobin count, mean corpuscular hemoglobin concentration and concomitant medications. The covariate model was established in a stepwise fashion. To compare hierarchical models, a likelihood ratio test was adopted. Changes in the objective function values (OFVs) caused by the inclusion of a covariate were proportional to twice the negative log likelihood of the data and approximated to a Chi-square distribution. In the univariate analysis, a decrease in the OFV $>3.84(\mathrm{P}<0.05$, degrees of freedom=1) was used as a criterion for inclusion of the covariate in the base model. The significant covariate-parameter associations were reserved in the model. When a full regression model was built, the model was further refined by eliminating the covariate from each parameter one at a time in order to acquire the final model. An increase in the OFV $>6.64(\mathrm{P}<0.01$, degrees of freedom=1) was used as a criterion for retaining significant covariate-parameter associations in the model (13).

Model validation. The goodness-of-fit plots of the final model was generated by the statistics package of $\mathrm{R}$ version 3.4.2 (https://www.r-project.org/). An internal validation method of bootstrap was used to evaluate the reliability and stability of the final parameter estimates. Bootstrap was generated by repeated random sampling, with replacement of the original data. This procedure was performed with the software package Wings for NONMEM and repeated 1,000 times with different random draws. The medians and $2.5-97.5 \%$ percentiles of the bootstrap result set parameters were compared with the final PK parameter estimates. The distribution of weighted residuals for the final model was evaluated by histograms and quantile-quantile plots (13).

\section{Results}

Data collection from real-world studies. A total of 18 Chinese pediatric patients with PRNS (13 males and 5 females) were available for population modeling. The patient characteristics and drug combinations are summarized in Tables I and II, respectively.

Modeling. A one-compartment model with first-order absorption and elimination was determined to be the most suitable model for the data. Ka was fixed to $0.68 / \mathrm{h}$, in accordance with a previously published study (9). The PK parameters of cyclosporin, CL/F 
Table I. Demographic and laboratory data of the subjects.

\begin{tabular}{|c|c|c|}
\hline Parameter & Mean \pm standard deviation & Median (range) \\
\hline Sex (male/female) & $13 / 5$ & l \\
\hline Age (years) & $2.79 \pm 0.90$ & $2.75(1.18-4.57)$ \\
\hline Weight (kg) & $15.28 \pm 2.95$ & $15(10-23)$ \\
\hline Height $(\mathrm{cm})$ & $91.28 \pm 8.23$ & $94(77-105)$ \\
\hline Albumin (g/l) & $19.68 \pm 6.82$ & $20.1(10.1-33.2)$ \\
\hline Globulin (g/l) & $24.69 \pm 3.18$ & $24.3(19.5-31.4)$ \\
\hline Albumin/globulin & $0.83 \pm 0.34$ & $0.75(0.4-1.7)$ \\
\hline Alanine transaminase (IU/l) & $12.12 \pm 12.71$ & $9(1-79)$ \\
\hline Aspartate transaminase (IU/l) & $26.35 \pm 15.84$ & $20(11-75)$ \\
\hline Creatinine $(\mu \mathrm{mol} / \mathrm{l})$ & $25.47 \pm 23.72$ & $20.5(7-152)$ \\
\hline Urea $(\mu \mathrm{mol} / \mathrm{l})$ & $4.19 \pm 2.05$ & $3.65(1.4-11.5)$ \\
\hline Total protein (g/l) & $45.16 \pm 7.41$ & $43.55(33.9-66.0)$ \\
\hline Total bile acid $(\mu \mathrm{mol} / \mathrm{l})$ & $4.79 \pm 3.85$ & $3.6(0.3-15.6)$ \\
\hline Direct bilirubin $(\mu \mathrm{mol} / \mathrm{l})$ & $0.81 \pm 0.83$ & $0.6(0.1-4.8)$ \\
\hline Total bilirubin $(\mu \mathrm{mol} / \mathrm{l})$ & $4.24 \pm 2.54$ & $3.5(1.3-12.1)$ \\
\hline Hematocrit (\%) & $39.64 \pm 5.17$ & $39(30.22-51.9)$ \\
\hline Hemoglobin (g/l) & $130.34 \pm 17.53$ & $127.5(97-173.1)$ \\
\hline Mean corpuscular hemoglobin (pg) & $25.66 \pm 3.31$ & $26.95(18-30)$ \\
\hline Mean corpuscular hemoglobin concentration (g/l) & $330 \pm 13.94$ & $333(301-353)$ \\
\hline
\end{tabular}

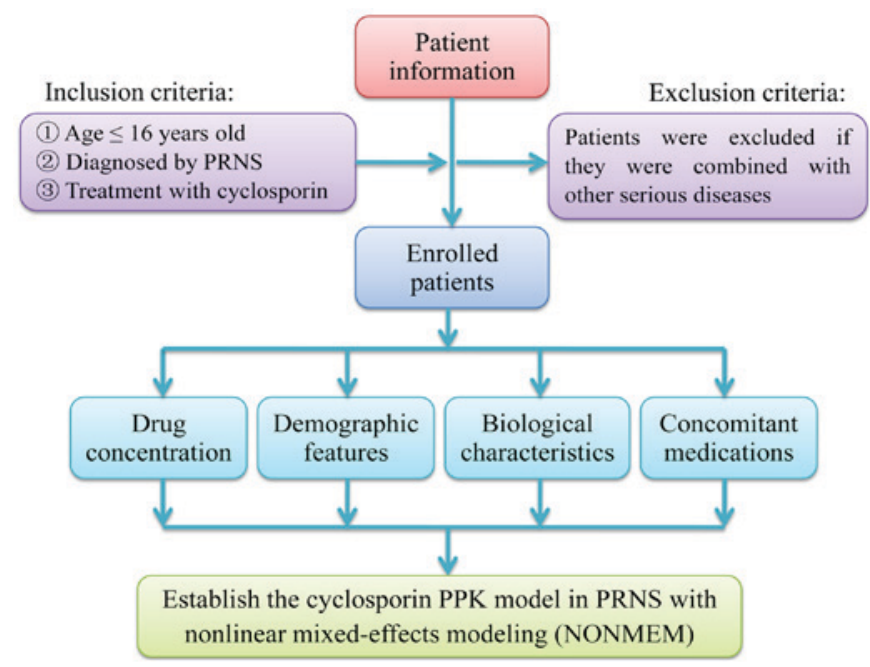

Figure 1. Schematic flow chart of the patient recruitment strategy $(n=18)$. PRNS, pediatric refractory nephrotic syndrome; PPK, population pharmacokinetics.

and $\mathrm{V} / \mathrm{F}$ were estimated using NONMEM. The final covariate models were as follows: $\mathrm{CL} / \mathrm{F}=\theta_{\mathrm{CL} / \mathrm{F}} \mathrm{x}(\mathrm{WT} / 70)^{0.75} \mathrm{x}(1+$ spirolactone $\left.\mathrm{x} \theta_{\text {spirolactone }}\right)$. Spirolactone may be 1 or 0 for yes and no, respectively regarding concomitant spirolactone administration; and $\mathrm{V} / \mathrm{F}=\theta_{\mathrm{V} / \mathrm{F}} \mathrm{x}(\mathrm{WT} / 70)$, where $\theta_{\mathrm{CL} / \mathrm{F}}$ and $\theta_{\mathrm{V} / \mathrm{F}}$ are the typical population values for $\mathrm{CL} / \mathrm{F}$ and $\mathrm{V} / \mathrm{F}$, respectively, and $\theta_{\text {spirolactone }}$ is the coefficient of spirolactone. The coefficient of spirolactone was negative.

Validation. The goodness-of-fit plots of the final model are provided in Fig. 2. Parameter estimates of the final model and bootstrap validation are provided in Table III. The median
Table II. Drug combinations with cyclosporin.

\begin{tabular}{|c|c|}
\hline Drug & $\mathrm{N}$ \\
\hline \multicolumn{2}{|c|}{ Diltiazem } \\
\hline No & 15 \\
\hline Yes & 3 \\
\hline \multicolumn{2}{|c|}{ Dipyridamole } \\
\hline No & 16 \\
\hline Yes & 2 \\
\hline \multicolumn{2}{|c|}{ Felodipine } \\
\hline No & 17 \\
\hline Yes & 1 \\
\hline \multicolumn{2}{|c|}{ Fosinopril } \\
\hline No & 15 \\
\hline Yes & 3 \\
\hline \multicolumn{2}{|c|}{ Methylprednisolone } \\
\hline No & 14 \\
\hline Yes & 4 \\
\hline \multicolumn{2}{|c|}{ Nifedipine } \\
\hline No & 17 \\
\hline Yes & 1 \\
\hline \multicolumn{2}{|c|}{ Piperazine ferulate } \\
\hline No & 13 \\
\hline Yes & 5 \\
\hline \multicolumn{2}{|c|}{ Prednisolone } \\
\hline No & 5 \\
\hline Yes & 13 \\
\hline \multicolumn{2}{|c|}{ Spirolactone } \\
\hline No & 7 \\
\hline Yes & 11 \\
\hline
\end{tabular}


Table III. Parameter estimates of final model and bootstrap validation.

\begin{tabular}{lccccc}
\hline & & & \multicolumn{2}{c}{ Bootstrap } \\
Parameter & Estimate & SE $(\%)$ & Median & 95\% Confidence interval & Bias (\%) \\
\hline $\mathrm{CL} / \mathrm{F}(\mathrm{L} / \mathrm{h})$ & 80.7 & 19.8 & 79.900 & $(53.1,106.5)$ & -0.991 \\
$\mathrm{~V} / \mathrm{F}(\mathrm{L})$ & 2030 & 75.4 & 2340 & $(687,3747.5)$ & 15.271 \\
$\mathrm{Ka}\left(\mathrm{h}^{-1}\right)$ & $0.68($ fixed) & - & - & - & - \\
$\theta_{\text {spirolactone }}$ & -0.265 & 33.4 & -0.266 & $(-0.448,-0.066)$ & 0.377 \\
$\omega_{\mathrm{CL} / \mathrm{F}}$ & 0.446 & 18.7 & 0.445 & $(0.201,0.569)$ & -0.224 \\
$\omega_{\mathrm{V} / \mathrm{F}}$ & 0.531 & 30.1 & 0.513 & $(0.005,0.749)$ & -3.390 \\
$\sigma_{1}$ & 0.117 & 18.4 & 0.115 & $(0.047,0.149)$ & -1.710 \\
$\sigma_{2}$ & 8.062 & 32.6 & 7.490 & $(0.212,11.843)$ & -7.095 \\
\hline
\end{tabular}

The 95\% confidential interval was displayed as the 2.5,97.5th percentile of bootstrap estimates. CL/F, apparent oral clearance (1/h); V/F, apparent volume of distribution (1); Ka, absorption rate constant (1/h); $\theta_{\text {spirolactone }}$, coefficient of the spirolactone; $\omega_{\mathrm{CL} / \mathrm{F}}$, inter-individual variability of $\mathrm{CL} / \mathrm{F} ; \omega_{\mathrm{V} / \mathrm{F}}$, inter-individual variability of $\mathrm{V} / \mathrm{F} ; \sigma_{1}$, residual variability, proportional error; $\sigma_{2}$, residual variability, additive error; Bias, prediction error, Bias $=($ Median-Estimate $) /$ Estimate $\mathrm{x} 100 \%$.
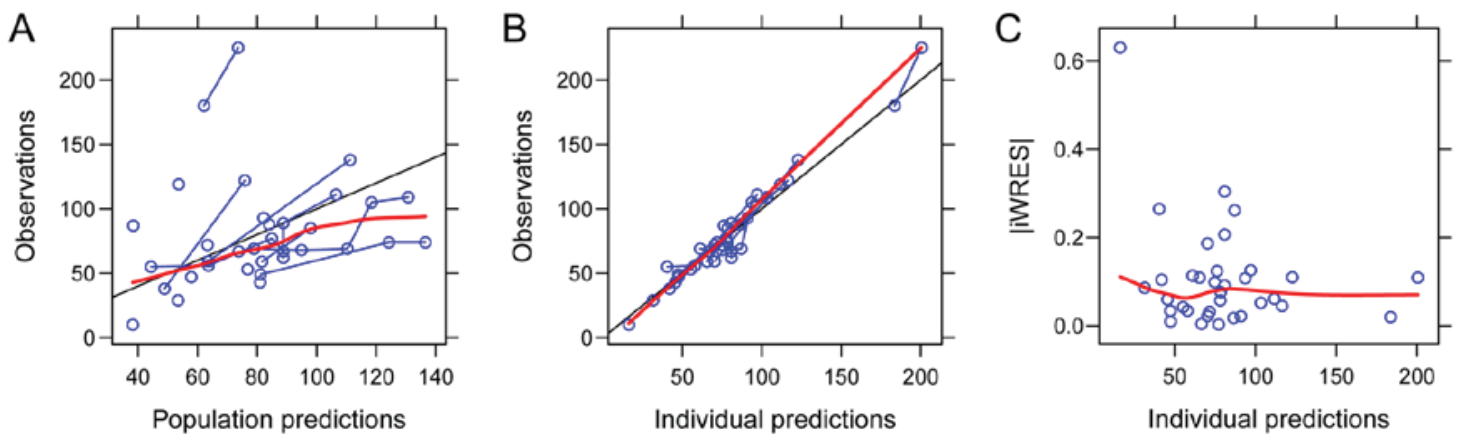

Figure 2. Goodness-of-fit plots of the final population model. (A) Observations vs. population predictions. (B) Observations vs. individual predictions. (C) Absolute values of iWRES vs. individual predictions. Black dashed lines, line of unity; Red smooth line, trend of data. iWRES, individual weighted residuals.
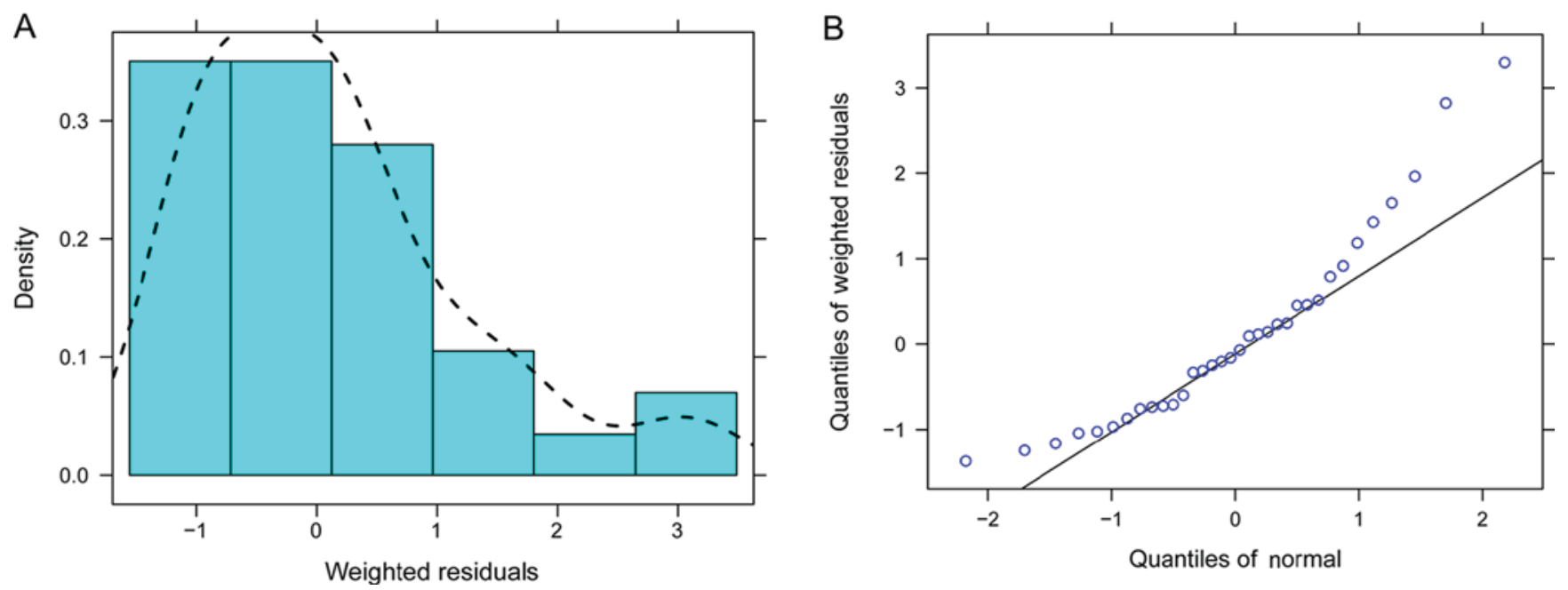

Figure 3. Distribution of weighted residuals for the final model. (A) Density vs. weighted residuals. (B) Quantiles of weighted residuals vs. normal quantiles.

values of the parameter estimate from bootstraps were close to the respective values from the final population model, demonstrating that the estimates for the PK parameters in the final population model were accurate and that the model was reliable. The distribution of weighted residuals in the final model is presented in Fig. 3. 


\section{Discussion}

The traditional therapy for treatment of PRNS has been steroids, although its long-term use may cause adverse effects on the growth and development of pediatric patients. Patients with PRNS undergo prolonged and repeated steroid therapy, which increases the risk of obesity, cushingoid appearance, osteoporosis, hypertension, infections, growth retardation and psychological problems (16). Cyclosporin has been widely used for patients with PRNS to improve treatment responses and to reduce the adverse reactions of steroid therapy (6-8). Since cyclosporin exhibits only a poor correlation between drug dosage and blood concentrations, PPK studies of cyclosporin have proven to be indispensable $(9,11,12)$.

To the best of our knowledge, the present study may be the first to provide a PPK model of cyclosporin in pediatric patients with PRNS. A cyclosporin PPK analysis was performed in Chinese PRNS patients using a population modeling approach, which was particularly suitable as a means of investigation, since logistical and ethical restrictions involved in studying pediatric patients prohibit excessive blood sampling compared with traditional PK studies (17). A cyclosporin PPK model with the ability to predict PK processes in individual patients with PRNS is of great value from a clinical perspective.

In the construction of the present model, a one-compartment model with first-order elimination was used to describe the absorption phase, since all the cyclosporin concentrations featured in the study were trough concentrations, and the Ka of the model was fixed to the value of $0.681 / \mathrm{h}$, according to that of a previous study (9). Typical values of $\mathrm{CL} / \mathrm{F}$ and $\mathrm{V} / \mathrm{F}$ in the final model were $80.7 \mathrm{l} / \mathrm{h}$ and 2,030 1, respectively. The inter-individual variabilities in $\mathrm{CL} / \mathrm{F}$ and $\mathrm{V} / \mathrm{F}$ were 44.6 and $53.1 \%$. An allometry-based population PK model was developed for cyclosporin: WT was incorporated, with allometric coefficients of 0.75 for $\mathrm{CL} / \mathrm{F}$ and 1 for $\mathrm{V} / \mathrm{F}$ (15). The present model also incorporated various covariates on different parameters, and the following covariate was determined to be meaningful: 'Spirolactone' on CL/F. The coefficient of spirolactone was negative, indicating that concomitant medication with spirolactone was able to reduce cyclosporin clearance in the patients with PRNS.

Of note, the present study had a number of limitations. First, pharmacogenomic considerations of spirolactone have not been verified in clinical use. Furthermore, the present study was a retrospective analysis based on real-world studies, which means that genotyping was not routinely performed in the study population. Whether the inclusion of genotyping in the present model may provide any improved explanation of the variabilities of spirolactone in PRNS should be studied in the future. In addition, it would be useful to evaluate the levels of T lymphocytes and/or the corresponding cytokines produced, indicating the release and concentration levels of the drug. However, this is not within the domain of PPK; such a study would belong to the domain of PK and pharmacodynamics (PD), and population $\mathrm{PD} / \mathrm{PK}$ of cyclosporin in patients with pediatric refractory nephrotic syndrome will be established in future studies.

In conclusion, to the best of our knowledge, the present study was the first to assess the PPK of cyclosporin in patients with PRNS, and the presence of a clinically significant interaction between spirolactone and cyclosporin in PRNS patients based on real-world studies was also investigated.
The coefficient of spirolactone was negative, indicating that concomitant medication with spirolactone was able to reduce cyclosporin clearance in the patients with PRNS.

\section{Acknowledgements}

Not applicable.

\section{Funding}

This work was supported by the Clinical Pharmacy Key Specialty Construction Project of Shanghai (grant no. YZ2017/5), the Young Medical Talents of Wuxi (grant no. QNRC020), the Young Project of Wuxi Health and Family Planning Research (grant no.Q201706), AOSAIKANG pharmaceutical foundation (grant no. A201826, the Wuxi Science and Technology Development Guidance Plan (Medical and Healthcare; grant. no. CSZON1744).

\section{Availability of data and materials}

The datasets used and/or analyzed during the current study are available from the corresponding author on reasonable request.

\section{Authors' contributions}

ZL and DW conceived and designed the study. DW and XC collected and analyzed data. DW and XC wrote the manuscript. All authors read and approved the final manuscript.

\section{Ethics approval and consent to participate}

The retrospective study was approved by the Research Ethics Committee of Children's Hospital of Fudan University (Shanghai, China). Written informed consent was not required, since the data were collected without patient identifiers.

\section{Patient consent for publication}

Not applicable.

\section{Competing interests}

The authors declare that they have no competing interests.

\section{References}

1. Eddy AA and Symons JM: Nephrotic syndrome in childhood. Lancet 362: 629-639, 2003.

2. McKinney PA, Feltbower RG, Brocklebank JT and Fitzpatrick MM: Time trends and ethnic patterns of childhood nephrotic syndrome in Yorkshire, UK. Pediatr Nephrol 16: 1040-1044, 2001

3. Koskimies O, Vilska J, Rapola J and Hallman N: Long-term outcome of primary nephrotic syndrome. Arch Dis Child 57: $544-548,1982$

4. Lombel RM, Gipson DS and Hodson EM; Kidney Disease: Improving Global Outcomes: Treatment of steroid-sensitive nephrotic syndrome: New guidelines from KDIGO. Pediatr Nephrol 28: 415-426, 2013.

5. Tarshish P, Tobin JN, Bernstein $\mathrm{J}$ and Edelmann CM Jr: Prognostic significance of the early course of minimal change nephrotic syndrome: Report of the international study of kidney disease in children. J Am Soc Nephrol 8: 769-776, 1997. 
6. Goumenos DS, Katopodis KP, Passadakis P, Vardaki E, Liakopoulos V, Dafnis E, Stefanidis I, Vargemezis V, Vlachojannis JG and Siamopoulos KC: Corticosteroids and ciclosporin A in idiopathic membranous nephropathy: Higher remission rates of nephrotic syndrome and less adverse reactions than after traditional treatment with cytotoxic drugs. Am J Nephrol 27: 226-231, 2007.

7. Kitano Y, Yoshikawa N, Tanaka R, Nakamura H, Ninomiya M and Ito $\mathrm{H}$ : Ciclosporin treatment in children with steroid-dependent nephrotic syndrome. Pediatr Nephrol 4: 474-477, 1990.

8. Tanaka R, Yoshikawa N, Kitano Y, Ito $\mathrm{H}$ and Nakamura $\mathrm{H}$ : Long-term ciclosporin treatmentin children with steroid-dependent nephrotic syndrome. Pediatr Nephrol 7: 249-252, 1993.

9. Ni SQ, Zhao W, Wang J, Zeng S, Chen SQ, Jacqz-Aigrain E and Zhao ZY: Population pharmacokinetics of ciclosporin in Chinese children with aplastic anemia: Effects of weight, renal function and stanozolol administration. Acta Pharmacol Sin 34: 969-975, 2013.

10. Vadcharavivad S, Praisuwan S, Techawathanawanna N, Treyaprasert W and Avihingsanon Y: Population pharmacokinetics of tacrolimus in Thai kidney transplant patients: Comparison with similar data from other populations. J Clin Pharm Ther 41: 310-328, 2016.

11. Fruit D, Rousseau A, Amrein C, Rollé F, Kamar N, Sebbag L, Redonnet M, Epailly E, Marquet P and Prémaud A: Ciclosporin population pharmacokinetics and Bayesian estimation in thoracic transplant recipients. Clin Pharmacokinet 52: 277-288, 2013.

12. Wilhelm AJ, de Graaf P, Veldkamp AI, Janssen JJ, Huijgens PC and Swart EL: Population pharmacokinetics of ciclosporin in haematopoietic allogeneic stem cell transplantation with emphasis on limited sampling strategy. Br J Clin Pharmacol 73: $553-563,2012$
13. Wang DD, Lu JM, Li Q and Li ZP: Population pharmacokinetics of tacrolimus in paediatric systemic lupus erythematosus based on real-world study. J Clin Pharm Ther 43: 476-483, 2018.

14. Chen Y, Wu D, Dong M, Zhu Y, Lu J, Li X, Chen C and Li Z: Population pharmacokinetics of vancomycin and AUC-guided dosing in Chinese neonates and young infants. Eur J Clin Pharmacol 74: 921-930, 2018

15. Anderson BJ and Holford NH: Mechanism-based concepts of size and maturity in pharmacokinetics. Annu Rev Pharmacol Toxicol 48: 303-332, 2008.

16. Hahn D, Hodson EM, Willis NS and Craig JC: Corticosteroid therapy for nephrotic syndrome in children. Cochrane Database Syst Rev: CD001533, 2015.

17. Kauffman RE and Kearns GL: Pharmacokinetic studies in paediatric patients. Clinical and ethical considerations. Clin Pharmacokinet 23: 10-29, 1992.

This work is licensed under a Creative Commons Attribution-NonCommercial-NoDerivatives 4.0 International (CC BY-NC-ND 4.0) License. 